\title{
Haemodynamically unstable pelvic trauma: initial validation of a dedicated protocol by a retrospective cohort study with historical controls
} \author{
CC Castelli, ${ }^{2}$ L Ansaloni ${ }^{1}$ \\ ${ }^{1}$ General Surgery I, Ospedale Papa Giovanni XXIII Bergamo Italy \\ ${ }^{2}$ Orthopaedics and Traumatology, Ospedale Papa Giovanni XXIII Bergamo Italy \\ ${ }^{3}$ Accident and Emergency Department, Ospedale Papa Giovanni XXIII Bergamo Italy \\ ${ }^{4}$ Intensive Care Unit, Ospedale Papa Giovanni XXIII Bergamo Italy
}

S Magnone, ${ }^{1}$ M Ceresoli, ${ }^{1}$ F Coccolini, ${ }^{1}$ GE Nita, ${ }^{1}$ L Rizzi, ${ }^{2}$ C Merli, ${ }^{3}$ R Colombi, ${ }^{3}$ D Piazzalunga, ${ }^{1}$ TM Valetti, ${ }^{4}$

Corresponding author: Stefano Magnone (smagnone@asst-pg23.it)

\begin{abstract}
Background: We present our experience after the introduction of Advanced Trauma Life Support (ATLS) ${ }^{\odot}$, Trauma Team (TT) and Preperitoneal Pelvic Packing (PPP) protocols for the treatment of hemodynamically unstable pelvic blunt trauma. Methods: This is a retrospective study with historical controls: before (Control Group, CG) and after (Study Group SG) the introduction of the protocol. A single physician managed the CG and angiography was the emergency manoeuvre. A team with ATLS guidelines and PPP as an emergency manoeuvre managed the SG. Data were collected retrospectively. Patients were divided into two groups: before and after the introduction of protocols.

Results: From January 2007 to October 2014, 36 patients were treated at our Centre. We consider patients from January 2007 to August 2011 (19 patients, CG) and from September 2011 to October 2014 (17 patients, SG). Median age was 54 years (43-69) in the CG vs. $47(40-63)$ in the SG $(\mathrm{p}=0.43)$, median initial SBP $90 \mathrm{mmHg}(85-103)$ in the CG 94 (69-103) in the SG, $(\mathrm{p}=0.60)$, heart rate was $90(80-110)$ in the CG and $110(95-130)$ in the SG, $(\mathrm{p}=0.09)$. Median Injury Severity Score was $33(21-41)$ in the CG and $34(26-41)$ in the SG $(p=0.29)$. Time from arrival in the Emergency Department to first therapeutic manoeuvre was 132 minutes $(109-180)$ in the CG and 87 minutes $(51-204)$ in the SG $(p=0.4)$. The difference in mortality was statistically significant: $64.7 \%(11 / 17)$ in the CG and $23.5 \%(4 / 17)$ in the SG $(\mathrm{p}=0.02)$.

Conclusions: The introduction of protocols changed our approach in hemodynamically unstable pelvic trauma, achieving a remarkable improvement in early mortality.

Keywords: preperitoneal pelvic packing, pelvic trauma, Advanced Trauma Life Support (ATLS) ${ }^{\circ}$, angiography, Trauma Team.
\end{abstract}

\section{Introduction}

Haemodynamically unstable pelvic trauma in multitrauma patients has been a challenge for years even in the most experienced Trauma Centres. Several management modalities have been proposed, but there is still no consensus on the appropriate algorithm. ${ }^{1-4}$ There are two opinions on this topic to control bleeding: primary use of angiography (AG) or emergent External Fixation (EF) of the pelvis and/ or Preperitoneal Pelvic Packing (PPP). AG was the first proposed in the 1970 s to control pelvic bleeding ${ }^{5,6}$ but a significant criticism of this approach is that pelvic fractures are more commonly associated with venous bleeding. ${ }^{7}$ AG has become the primary tool in haemorrhage control in the USA, ${ }^{8-10}$ even if a recent paper by Tesoriero et al. questioned this traditional approach. ${ }^{11}$ On the contrary, in Europe a different method, namely PPP, was proposed by Tim Pohlemann in $1994^{12}$ and Ertel in 2001. ${ }^{13}$ This approach consists of the control of the venous bleeding using pelvic retroperitoneal gauzes. $\mathrm{EF}$ and eventually $\mathrm{AG}$ are associated with PPP as needed if the patient does not improve. The World Society of Emergency Surgery published recent guidelines with suggestion towards this approach. ${ }^{14}$ The time-consuming activation of the Angio suite, which frequently relies on on-call personnel during off-hours and the difficult management of this kind of patient in an unsafe environment such as the Angio suite prompted us to evaluate 
the Denver experience after a one month visit in 2011., , $^{2-16}$ On this basis, we developed a new algorithm for our hospital that was later validated during a national consensus conference. ${ }^{17}$ This paper aims to present our experience in early ( 24 hours) mortality of unstable pelvic trauma before and after the introduction of the Trauma Team (TT) with ATLS ${ }^{\circledR}$ guidelines and a dedicated protocol. We hypothesised that these three interventions contributed to lower first 24 hours mortality for pelvic bleeding.

\section{Methods}

A retrospective analysis with historical controls was conducted to validate a new protocol for the treatment of haemodynamically unstable pelvic adult trauma patients evaluated at our institution from January 2007 to October 2014. Data were retrieved from the administrative database of our hospital according to the International Classification of Diseases (ICD-9 CM). Patients coding 808.0-808.9 concerning open or closed pelvic or acetabular fractures as a first or second diagnosis were considered. The retrieved file was then filtered by manual revision of all clinical notes considering only patients with haemodynamic instability (defined as Systolic Blood Pressure (SBP) $<90 \mathrm{mmHg}$ despite appropriate resuscitation as clinical judgement according to the Trauma Team) and a pelvic and/or acetabular fracture. Acetabular fractures were considered because they are sometimes responsible for haemodynamic instability due to arterial damage requiring angioembolization. ${ }^{18-19}$ Fractures were classified retrospectively by one of the authors (SM) according to ArbeitsgemeinschaftfürOsteosynthesefrag en-American Orthopaedic Trauma Association (AO-OTA) classification, namely pelvic fractures were classified as A (lesion sparing (or with no displacement of) posterior arch), B (incomplete disruption of posterior arch, partially stable) or $\mathrm{C}$ (complete disruption of posterior arch, unstable). ${ }^{20}$ Our hospital has been designated Level I Trauma Centre. It is a one thousand beds general hospital with a catchment area around 1.2 million people. In 2011, a significant internal change occurred in the management of trauma. A Trauma Team (TT) consisting of a general surgeon, an emergency physician and an anesthesiologist was established, together

\section{Haemodynamically UNSTABLE PELVIC TRAUMA ALGORYTHM}

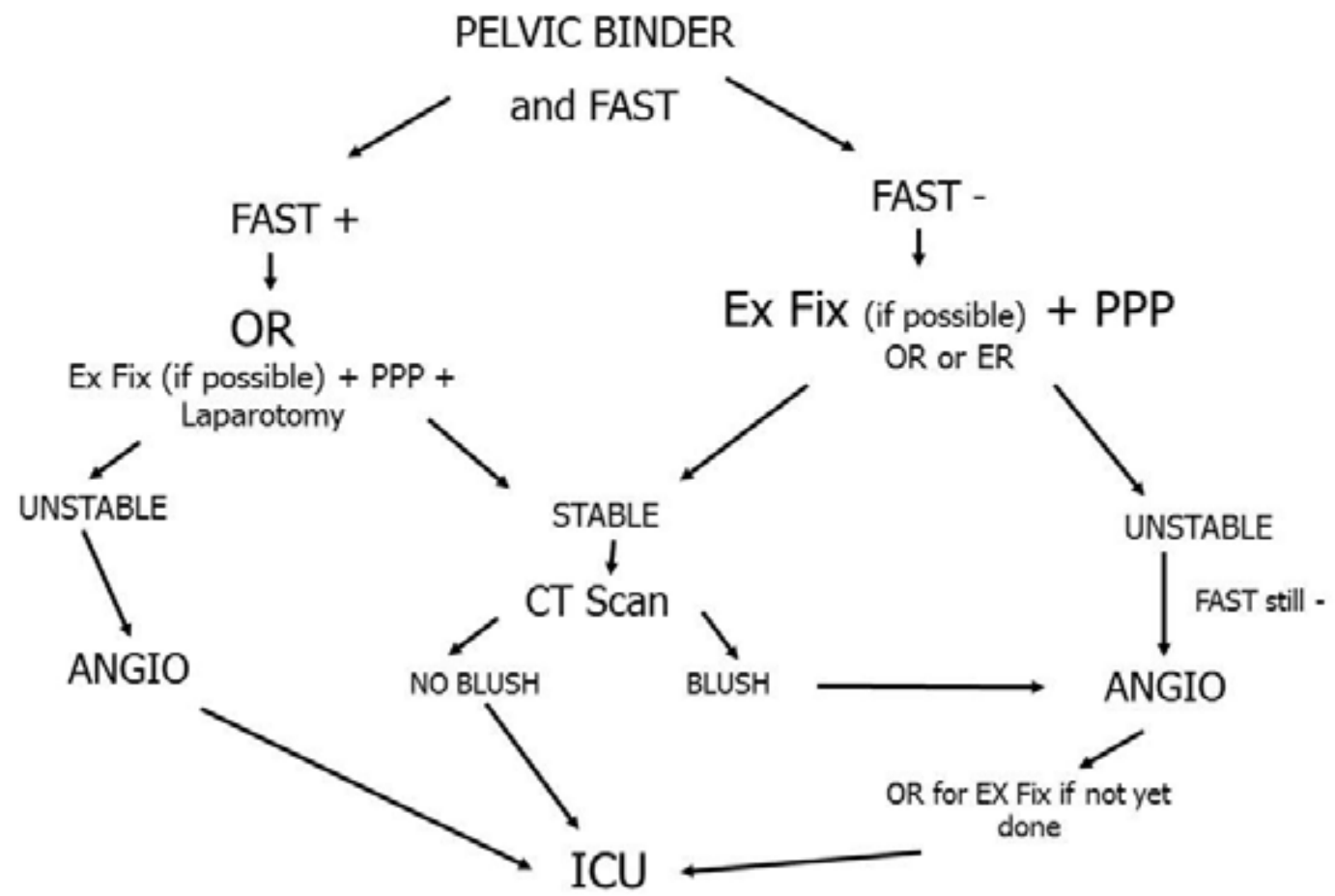

Figure.1 Treatment algorithm

Legend

FAST: focused assessment sonography for trauma

PPP: preperitoneal pelvic packing

OR: operating room

Ex Fix: external fixation

ER: emergency room 
with ATLS $®$ guidelines introduction. The Emergency Medical System from the scene of trauma prompts TT activation, and the TT treats the patients from arrival in the ED. In the absence of the TT, treatment options were agreed on between the physician in charge of the patient (namely an anesthesiologist) and the surgeon or orthopaedic as referrals; there was no team decision. PPP was not in use and AG was the only therapeutic tool when promptly feasible. The new protocol (Figure 1) was established in September 2011 and is based on PPP and EF, whenever possible and achievable, of haemodynamically unstable pelvic trauma. For both groups, no massive transfusion protocol was in use.

Patients were divided into two groups: before and after the introduction of ATLS ${ }^{\circledR}$ guidelines, Trauma Team and Preperitoneal Pelvic Packing (August 2011). The historical Control Group (CG) was identified from September 2007 to August 2011 while the Study Group (SG) comprises patients treated from September 2011 to October 2014. All patients had a temporary stabilisation with a pelvic sheet (either homemade or commercially available). The Injury Severity Score (ISS) was calculated by two authors independently and in case of disagreement a third author made the final decision. Information regarding organ lesions come from CT scan, $\mathrm{X}$ ray, ultrasound or intraoperative findings. The two groups were compared to ensure a homogeneous population. A subgroup analysis of the pelvic fractures, with the exclusion of the acetabular fractures, was conducted. Continuous data are expressed as median (and Interquartile Range IQR) or mean (and standard deviation SD) as appropriate and were compared with the Mann-Whitney test for non-normally distributed variables or Student t-test for normally distributed. Categorical data were expressed as proportions and percentages and were compared with the Pearson uncorrected test. $\mathrm{P}<0.05$ was assumed as significant. Statistics were calculated using SPSS 20.0 (IBM Corp. Released 2011. IBM SPSS Statistics for Windows, Version 20.0. Armonk, NY: IBM Corp).

\section{Results}

From January 2007 to October 2014, 36 patients were treated in our Centre and considered in this paper. Nineteen patients were included in the Control Group and 17 patients in the Study Group. The two groups were compared for age, sex, initial $\mathrm{SBP}$, heart rate (HR), $\mathrm{pH}$, base excess haemoglobin level $(\mathrm{Hb})$ and ISS and did not result significantly different. Serum lactate level was not available in our ED until 2012. On the other hand, fibrinogen level, International Normalized Ratio (INR) and platelets level did not differ between groups. (Table 1). The median length of stay in the Emergency Department (ED) was 83 minutes (39-151) in the SG and 161 minutes $(114-243)$ in the CG $(p=0.02)$. Twelve patients $(63.2 \%)$ in the CG underwent a therapeutic procedure (AG or surgery) while in the SG all 17 patients underwent a procedure $(\mathrm{p}=0.005)$. In the CG $5(26.3 \%)$ patients had positive FAST and underwent a surgical procedure (four underwent laparotomy with three splenectomies, and one intraperitoneal pelvic packing and one more patient underwent EF of a femur and tibia) and seven (36\%) underwent AG. Patients who underwent AG were all embolised, but 3 out of 7 patients died of pelvic bleeding after AG. In the SG all 17 patients underwent a procedure (15 patients underwent PPP and eight underwent laparotomy

Table 1. Characteristics of patients

\begin{tabular}{|c|c|c|c|}
\hline & Control Group $(1 / 2007-8 / 2011) n=19$ & Study group $(9 / 2011-10 / 2014) \mathrm{N}=17$ & $\mathbf{p}$ \\
\hline Age & $54(43-69)$ & $47(40--63)$ & 0.43 \\
\hline $\operatorname{Sex}(M / F)$ & $15 / 4$ & $12 / 5$ & 0.56 \\
\hline Initial SBP & $90(85-103)$ & $94(69-103)$ & 0.60 \\
\hline Patients with $\mathrm{SBP}<90$ & $4(21.1 \%)$ & $5(29.4 \%)$ & 0.56 \\
\hline Heart Rate & $90(80-110)$ & $119(95-130)$ & 0.09 \\
\hline $\mathrm{pH}$ & $7.09(7.05-7.29)$ & $7.21(7.20-7.27)$ & 0.92 \\
\hline $\mathrm{BE}$ & $-11.8(-12--5.0)$ & $-8.0(-10.0--5.0)$ & 0.64 \\
\hline Haemoglobin (g) & $9.0(7.1-11.1)$ & $11.0(10.0-11.5)$ & 0.22 \\
\hline Fibrinogen & $141(88-183)$ & $116(57-160)$ & 0.47 \\
\hline Platelets & $145500(85750-201750)$ & $171000(101000-242000)$ & 0.51 \\
\hline INR & $1.48(1.25-1.85)$ & $1.48(1.32-1.97)$ & 0.93 \\
\hline Injury Severity Score & $33(21-41)$ & $34(26-41)$ & 0.29 \\
\hline \multicolumn{4}{|l|}{ Type of pelvic fracture } \\
\hline $\mathrm{A}^{*}$ & $4(21 \%$, one acetabular $)$ & 0 & na \\
\hline B & $7(37 \%)$ & $9(53 \%)$ & 0.33 \\
\hline $\mathrm{C}$ & $7(37 \%)$ & $8(47 \%)$ & 0.53 \\
\hline
\end{tabular}


Table 2. Results in the two groups

\begin{tabular}{|c|c|c|c|}
\hline & Control Group (1/2007-8/2011) & Study group $(9 / 2011-10 / 2014)$ & $\mathbf{p}$ \\
\hline ED LoS (IQR min) & $161(114-243)$ & $39(39-151)$ & 0.02 \\
\hline All procedures & $12(63 \%)$ & $17(100 \%)$ & 0.005 \\
\hline \multirow[t]{3}{*}{ Surgical procedures } & $5(26.3 \%)$ & $8(47 \%)$ & 0.19 \\
\hline & $\begin{array}{c}4 \text { laparotomies (3 splenectomies and one } \\
\text { intraperitoneal packing) }\end{array}$ & $\begin{array}{l}8 \text { laparotomies and one thoracotomy } \\
\qquad 4 \mathrm{EF}\end{array}$ & \\
\hline & $1 E F$ & & \\
\hline Angiography & 7 (7 embolizations, $100 \%$ ) & 10 (4 embolizations, $40 \%$ ) & 0.01 \\
\hline PPP & 0 & 15 & \\
\hline Time to intervention* & $132(109-180)$ & $87(51-204)$ & 0.4 \\
\hline Mortality & $63.2 \%(12 / 19)$ & $23.5 \%(4 / 17)$ & 0.017 \\
\hline Packed red blood cells (units) & $8.0(4.0-14.0)$ & $10.5(5.5-16.8)$ & 0.18 \\
\hline Fresh Frozen Plasma (units) & $5.0(4.3-7.8)$ & $9.0(5.0-11.5)$ & 0.31 \\
\hline Crystalloids (ml) & $1500(875-2250)$ & $1250(1000-2375)$ & 0.85 \\
\hline
\end{tabular}

with a separate incision, one anterolateral thoracotomy, and four EF: three anterior fixations and one with a C clamp). Only 3 patients had positive FAST. During laparotomy, we recorded one splenectomy, one bowel resection, a mesenteric laceration and a liver trauma. Intraperitoneal packing was done in two patients, and in four cases laparotomy in the SG did not conduct to any therapeutic procedure (two patients had a negative FAST). Of the 15 patients who had a PPP, 10 underwent AG because of permanent haemodynamic instability (4 with successful therapeutic embolisation, 40\%) subsequently. One patient underwent a negative $A G$ and then was sent to the Intensive Care Unit. Another patient had a haemodynamic improvement with pelvic EF only. The median length of stay in the Emergency Department (ED) was 83 minutes (39-151) in the SG and 161 minutes $(114-243)$ in the CG $(\mathrm{p}=0.02)$. Median time from arrival in the ED to the first intervention was 87 minutes (51-204) in the SG vs 132 minutes (109-180) in the CG, $(\mathrm{p}=0.42)$. In three cases, there was a misleading delay due to the unavailability of the operating room, wrong clinical judgment or incorrect triage (253, 952 and 313 minutes respectively). Considering only emergent PPP median time to intervention in the SG (12 patients) was 61 minutes (54-105) which was significantly shorter $\mathrm{p}=0.04$.

Mortality in the first 24 hours was significantly higher in the CG: $63.2 \%(12 / 19)$ vs $23.5 \%(4 / 17), p=0.004$. In the $\mathrm{CG}$ all patients but one died because of pelvic bleeding: 8 patients did not undergo any procedure, 3 died after $A G$ and one because of severe traumatic brain injury. No one patient in the SG died from persistent pelvic bleeding. One death occurred for severe traumatic brain injury and three for acute traumatic coagulopathy and diffuse bleeding. PPP was removed after a median of 2 days (2-4) (Table 2). Transfusion and infusion rate were not significantly different between the two groups (Table3). We observed in the SG, among patients where PPP was carried out, two cases of sciatic nerve roots lesion. Other complications in the PPP group were two wound infections (26.6\% overall complication rate related to PPP). In the subgroup with only pelvic fractures without acetabular lesions, the CG accounted for 17 patients as did the SG. Mortality was $64.7 \%(11 / 17)$ in the $\mathrm{CG}$ and $23.5 \%(4 / 17)$ in the SG $(\mathrm{p}=0.017)$.

\section{Discussion}

Optimal treatment for haemodynamically unstable pelvic trauma is still under debate. There are two major approaches: AG first or PPP first. AG is still regarded as the primary tool in many US Trauma Centres, ${ }^{1,4}$ despite recent rethinking of the approach. ${ }^{11}$ PPP has been an option for the last two decades only, mainly in European trauma centres. ${ }^{21}$ In our hospital, a surgeon with interest in trauma is in 24-hours-a-day while the interventional radiologists are on call during off-hours. All this background and the Denver experience ${ }^{2,15-16}$ prompted our group to implement an algorithm in 2011 with PPP as the first surgical manoeuvre in case of haemodynamic instability and a pelvic fracture (Figure 1). We believe our previous experience, with AG as the first approach, could have delayed treatment due to the time-consuming activation of the interventional radiologic suite, as in other recent experience. ${ }^{22}$ Previous data demonstrated that $\mathrm{AG}$ is far more time consuming than PPP, ${ }^{23-25}$ so we preferred to consider PPP as the first and quickest choice for treatment, with $A G$ as a second option in case of permanent haemodynamic instability. AG was not achieved in many patients in the $\mathrm{CG}$ due to the longer time to prepare for the procedure, especially during the night and on weekends.

EF is considered an essential manoeuvre in the management of these patients and our protocol implemented it when feasible. This paper is the first presentation of our results of threeyears' experience. We noted a dramatic improvement in mortality and a shorter time managing these challenging 
patients in the first hours since arrival in the ED. We did not find an explanation for the four patients with an A-type of fracture in the CG. In the SG, only B-and C-type of fractures were encountered, but we considered it negligible for our analysis because type A fractures are considered less severe compared to B- and C-type, and results were better in the SG. Moreover, the type of fracture is not considered a risk factor for mortality in a multivariate analysis on 5014 patients. ${ }^{26} \mathrm{~A}$ possible explanation could be a better attitude to intensive resuscitation both in the out and the in-hospital setting that has been developing since we started the Trauma Centre program so that a prompt resuscitation led to exclude type A fracture patients from the haemodynamically unstable group. Nevertheless, the overall impact of ATLS $®$ and Trauma Team cannot be overemphasised nor undervalued..$^{27}$ Even in the most experienced Trauma Centres, AG needs a long time to be activated, and this always leads to very late treatment. ${ }^{11} \mathrm{We}$ believe that patients' management by a multidisciplinary team reduced ED length of stay and time to intervention and allowed better treatment for all the patients. ${ }^{28}$ Moreover a more aggressive resuscitation with blood components, even if not statistically significant could play a role, but we think that a significant role was played by PPP introduction as in other experience ${ }^{29-30}$ and review. ${ }^{31-33}$ In two cases, emergent EF of the pelvis, either by a C-clamp as first described by $\mathrm{Ganz}^{34}$ or by an anterior fixation, provided bleeding control without the need for PPP. It takes a considerable effort to achieve an optimal collaboration in a multidisciplinary team because we don't as yet have a fully committed orthopaedics' team and in some cases we had to force a PPP with pelvic binder as a substitute only because we could not obtain an emergent EF. This issue is part of continuous professional development since we are aware of the importance of EF in the management of these complex patients. Our algorithm is based on PPP with concomitant EF as the first therapeutic approach to haemodynamically unstable pelvic trauma, but we have realised, during these first three years, that a closer collaboration with the orthopaedic team is necessary for a correct implementation of the clinical pathway. We realised that more than twenty orthopaedic surgeons in the emergency rota are a critical issue to gain confidence with such emergent decisions. Our experience differs from others', which can more easily involve orthopaedic surgeons to fix the pelvis and think that PPP can be harmful instead of beneficial for the haemodynamic status of these patients; however, we are not in the position to agree with that statement..$^{35}$

PPP usually demands a midline vertical or, less frequently, horizontal incision. The vertical one could be a problem for orthopaedics during the operating room internal fixation of the pubis, mainly if packing must be repeated more times. We do not believe that pelvic binder alone is sufficient to control dramatic bleeding from cancellous bone and venous vessels damage, due to the temporary effect and the fear of skin pressure sores. ${ }^{36-39}$ So we suggest, as other trauma centres, EF and/or PPP as the best emergent treatment for these patients..$^{2,15-16,40}$ One argument against our conclusion could be that the significant impact on mortality was by ATLS and TT rather than PPP, because in all our trauma population, excluding severe (AIS > 2) traumatic brain injury, mortality improved in the SG. ${ }^{26}$ This statement is not correct in our opinion because in the CG surgical procedures did not address pelvic bleeding but only intraperitoneal one. Moreover, AG was successful in $7 / 7$ patients, but 3 of them died as well, while the SG needed angioembolization only in four patients, as in the six remaining patients, AG was negative after PPP. The two groups were statistically different in terms of embolization rate: we have no particular explanation but the fact that a more aggressive surgical bleeding control with PPP gives the chance to spare a higher number of IR procedures. The lower rate of embolization in the SG is probably due to an aggressive attitude to angiography after PPP which is not warranted. We are confident that gaining experience in correct resuscitation will lead to more appropriate IR use. Not surprisingly, we found a 1:1 ratio for PRBC/Fresh Frozen Plasma in the SG, versus a 1:0.5 in the $\mathrm{CG}$; crystalloids infusion did not differ so much in the two groups. For both groups, no massive transfusion protocol was in use. None of these differences are statistically significant, but this could have a partial effect on the improvement of mortality. ${ }^{41}$ Other authors consider the appropriate coagulation management the only factor to achieve bleeding control, ${ }^{42}$ but in our experience PPP is the surgical procedure that gained statistical significance and allowed the steep improvement in these patients, notwithstanding the importance of the TT and ATLS approach. Moreover, in both groups trauma-induced coagulopathy did not differ, so patients in the CG did not have an inadequate resuscitation, as one could speculate based on PRBC/FFP ratio or the amount of transfusion patients received. In our experience, we found a high incidence of no therapeutic laparotomy $(4 / 8,50 \%)$. We believe that in the initial phase of our experience of patient management, profound shock could play a role in the decision to access the peritoneal cavity despite a negative FAST. Another interesting finding is that patients who underwent AG after PPP had a lower incidence of embolization ( $40 \%$ instead of $100 \%)$. A possible explanation is that in the CG AG was the only method to control bleeding, while in the SG it comes after PPP and so most of the haemostasis has already been achieved even for small arterial vessels. At the beginning of our experience AG may have been regarded as a mere completion of the surgical treatment, perhaps providing a reason of the high negative rate of embolization. In the future, a closer insight into the correct resuscitation process could avoid unnecessary interventional manoeuvres. Major arterial bleeding, on the other hand, mandates control as soon as possible. We must report two sciatic roots damage in the early phase of our PPP experience that was not previously reported in the trauma literature. ${ }^{43}$ These two patients had a long-lasting postoperative course with a complete recovery only after one to two years. We are not in the position to exclude that this kind of lesion was due to the PPP technique rather than a sacral fracture/dislocation. On the other hand, we believe that too tight packing could be the cause of this problem at the beginning of our experience and we modified our 
packing technique now using smaller pads. However, major complications directly due to the procedure seem to be low and, together with the encouraging results, this treatment should be the first choice in case of haemodynamic instability. Our work has several limitations: it is a single centre study, even if the vast majority of reports on this topic come from single centres; data are retrospective and were retrieved from an administrative registry with a possible loss of some patients. Moreover, statistical significance is often very close to significance, but we think this is due to the small number of included patients. Another possible selection bias could be due to the creation of the regional trauma system, which from April 2011 had a significant impact on centralisation of patients. The new trauma system could have an impact on the numbers of severe trauma cases in our hospital, leading to a significant commitment of the TT, and a more expeditious therefore a better experience because of a higher caseload could have influenced the results. It is hard to assess the contribution of each modification in the treatment protocol because we know that TT and ATLS guidelines help to improve survival of patients. In the American setting, AG first is still regarded as the best option for emergency bleeding, even if arterial bleeding has been accounted only for $15 \%$ of cases. ${ }^{7}$ On the other hand, we think the PPP has added a significant part to the lifesaving procedure ${ }^{2,15-16,44}$ because AG needs to be more settled, at least in our experience, even if other groups reported longer time to prepare to favour PPP. ${ }^{45-}$ ${ }^{47} \mathrm{On}$ the other hand the availability of in-house Angio service 24 hours a day is far from being widespread., ${ }^{2,46} \mathrm{EF}$ is of paramount importance, but in our experience, its role is far from being improved.

\section{Conclusion}

The introduction of ATLS ${ }^{\circ}$ guidelines, Trauma Team and Preperitoneal Pelvic Packing changed our approach in haemodynamically unstable trauma patients, with a better trend regarding early mortality, time spent in the ED and time to the first therapeutic procedure. Major complication rate seems to be acceptable, and the efficacy of bleeding control demonstrated dramatic effects. We need further study to confirm these first favourable data.

\section{REFERENCES}

1. Cullinane DC, Schiller HJ, Zielinski MD, Bilaniuk JW, Collier BR, Como J, et al. Eastern Association for the Surgery of Trauma practice management guidelines for haemorrhage in pelvic fracture - update and systematic review. J Trauma. 2011;71(6):1850-68.

2. Burlew CC, Moore EE, Smith WR, Johnson JL, Biffl WL, Barnett CC, et al. Preperitoneal pelvic packing/external fixation with secondary angioembolization: optimal care for lifethreatening hemorrhage from unstable pelvic fractures. J Am Coll Surg. 2011;212(4):628-35(Discussion 635-7).

3. Burlew CC, Moore EE, Stahel PF, Geddes AE, Wagenaar AE, Pieracci FM, et al. Preperitoneal pelvic packing reduces mortality in patients with life-threatening hemorrhage due to unstable pelvic fractures. J Trauma Acute Care Surg. Feb 2017;82(2):233-42.
4. Davis JW, Moore FA, McIntyre RC, Cocanour CS, Moore EE, West MA.Western Trauma Association critical decisions in trauma: management of pelvic fracture with haemodynamic instability. J Trauma. 2008;65:1012-5.

5. Margolies MN, Ring EJ, Waltman AC, Kerr WS, Baum S.Arteriography in the management of hemorrhage from pelvic fractures. N Engl J Med. 1972;287:317-21.

6. Matalon TS, Athanasoulis CA, Margolies MN, Waltman AC, Novelline RA,Greenfield AJ, et al. Hemorrhage with pelvic fractures: efficacy of transcatheter embolization. Am J Roentgenol. 1979;133:859-64.

7. Huittinen VM, Slatis P. Postmortem angiography and dissection of the hypogastric artery in pelvic fractures. Surgery. 1973;73:454-62.

8. Morozumi J, Homma H, Ohta S, Noda M, Oda J, Mishima S, et al. Impact of mobile angiography in the emergency department for controlling pelvic fracture hemorrhage with haemodynamic instability. J Trauma. 2010;68:90-5.

9. Panetta T, Sclafani SJ, Goldstein AS, Phillips TF, Shaftan GW. Percutaneous transcatheter embolization for massive bleeding from pelvic fractures. J Trauma. 1985;25:1021-9.

10. Piotin M, Herbreteau D, Guichard JP, Houdart E, Reizine D, Aymard A, et al. Percutaneous transcatheter embolization in multiply injured patients with pelvic ring disruption associated with severe hemorrhage and coagulopathy. Injury. 1995;26:67780.

11. Tesoriero RB, Bruns BR, Narayan M, Dubose J, Guliani SS, Brenner ML, et al. Angiographic embolization for hemorrhage following pelvic fracture: Is it "time" for a paradigm shift? J Trauma Acute Care Surg. Jan 2017;82(1):18-26.

12. Pohlemann T, Gansslen A, Bosch U, Tscherne U. The technique of packing for control of hemorrhage in complex pelvic fractures. Tech Orthop. 1994,9:267-70.

13. Ertel W, Keel M, Eid K, Platz A, Trentz O. Control of severe hemorrhage using C-clamp and pelvic packing in multiply injured patients with pelvic ring disruption. J Orthop Trauma. 2001115:468-74.

14. Coccolini F, Stahel PF, Montori G, Biffl W, Horer TM, Catena F, et al. Pelvic trauma: WSES classification and guidelines. World J Emerg Surg. 18 Jan 2017;12:5.

15. Smith WR, Moore EE, Osborn P, Agudelo JF, Morgan SJ, Parekh AA, et al. Retroperitoneal packing as a resuscitation technique forhemodinamically unstable pelvic fractures: report of two cases and description of technique. J Trauma. 2005;59:1510-4.

16. Cothren CC, Osborn PM, Moore EE, Morgan SJ, Johnson JL, Smith WR. Preperitoneal pelvic packing for haemodynamically unstable pelvic fractures: a paradigm shift. J Trauma. 2007;62:834-42.

17. Magnone S, Coccolini F, Manfredi R, Piazzalunga D, Agazzi $\mathrm{R}$, Arici $\mathrm{C}$ et al Management of haemodynamically unstable pelvic trauma: results of the first Italian consensus conference (cooperative guidelines of the Italian Society of Surgery, the Italian Association of Hospital Surgeons, the Multi-specialist Italian Society of Young Surgeons, the Italian Society of Emergency Surgery and Trauma, the Italian Society of Anesthesia, Analgesia, Resuscitation and Intensive Care, the Italian Society of Orthopedics and Traumatology, the Italian Society of Emergency Medicine, the Italian Society of Medical Radiology -Section of Vascular and Interventional Radiologyand the World Society of Emergency Surgery). World J Emerg Surg. 2014 Mar 7;9(1):18

18. Pascarella R, Del Torto M, Politano R, Commessatti M, Fantasia 
R, Maresca A. Critical review of pelvic fractures associated with external iliac artery lesion: a series of six cases. Injury. Feb 2014;45(2):374-8.

19. Lindahl J, Handolin L, Söderlund T, Porras M, Hirvensalo E. Angiographic embolization in the treatment of arterial pelvic hemorrhage: evaluation of prognostic mortality-related factors. Eur J Trauma Emerg Surg. Feb 2013;39(1):57-63.

20. Marsh JL, Slongo TF, Agel J, Broderick JS, Creevey W, DeCoster TA, et al. Fracture and dislocation classification compendium - 2007: Orthopaedic Trauma Association classification, database and outcomes committee. J Orthop Trauma. Nov-Dec 2007;21(Suppl 10):S1-133.

21. Papakostidis C, Giannoudis PV. Pelvic ring injuries with haemodynamic instability: efficacy of pelvic packing, a systematic review. Injury. 2009;40(Suppl 4):S53e61.

22. Schwartz DA, Medina M, Cotton BA, Rahbar E, Wade CE, Cohen AM, et al. Are we delivering two standards of care for pelvic trauma? Availability of angioembolization after hours and on weekends increases time to therapeutic intervention. J Trauma Acute Care Surg. Jan 2014;76(1):134-9.

23. Hauschild O, Aghayev E, von Heyden J, Strohm PC, Culemann $\mathrm{U}$, Pohlemann T, et al. Angioembolization for pelvic hemorrhage control: results from the German pelvic injury register. J Trauma Acute Care Surg. Sep 2012;73(3):679-84.

24. Fang JF, Shih LY, Wong YC, Lin BC, Hsu YP. Repeat transcatheter arterial embolization for the management of pelvic arterial hemorrhage. J Trauma. Feb 2009;66(2):429-35.

25. Tötterman A, Dormagen JB, Madsen JE, Kløw NE, Skaga NO, Røise O. A protocol for angiographic embolization in exsanguinating pelvic trauma: a report on 31patients. Acta Orthop. Jun 2006;77(3):462-8.

26. Pohlemann T, Stengel D, Tosounidis G, Reilmann H, Stuby F, Stöckle U, et al. Survival trends and predictors of mortality in severe pelvic trauma: estimates from the German Pelvic Trauma Registry Initiative. Injury. Oct 2011;42(10):997-1002.

27. Magnone S, Allegri A, Belotti E, Castelli CC, Ceresoli M, Coccolini $\mathrm{F}$, et al. Impact of ATLS guidelines, trauma team introduction, and 24-hour mortality due to severe trauma in a busy, metropolitan Italian hospital: A case control study. Ulus Travma Acil Cerrahi Derg. May 2016;22(3):242-6.

28. Tiel Groenestege-Kreb D, van Maarseveen O, Leenen L. Trauma team. Br J Anaesth. Aug 2014;113(2):258-65.

29. Lustenberger T, Meier C, Benninger E, Lenzlinger PM, Keel MJ. C-clamp and pelvic packing for control of hemorrhage in patients with pelvic ring disruption. J Emerg Trauma Shock. Oct 2011;4(4):477-82.

30. Abrassart S, Stern R, Peter R. Unstable pelvic ring injury with haemodynamic instability: what seems the best procedure choice and sequence in the initial management? Orthop Traumatol Surg Res. Apr 2013;99(2):175-82.

31. Gansslen A, Hildebrand F, Pohlemann T. Management of haemodynamic unstable patients "in extremis" with pelvic ring fractures. Acta chirurgiae orthopaedicae et traumatologiae Cechoslovaca. 2012;79:193-202.

32. Gaarder C, Naess PA, Frischknecht Christensen E, Hakala P, Handolin L, Heier HE, et al. Scandinavian Guidelines "The massively bleeding patient". Scandinavian Journal of Surgery
(SJS): Official Organ for the Finnish Surgical Society and the Scandinavian Surgical Society. 2008;97:15e36.

33. Tosounidis TI, Giannoudis PV. Pelvic fractures presenting with haemodynamic instability: treatment options and outcomes. Surgeon. Dec 2013;11(6):344-51.

34. Ganz R, Krushell RJ, Jakob RP, Küffer J. The antishock pelvic clamp. Clin Orthop. 1991;267:71-8.

35. Tonetti J. Management of recent unstable fractures of the pelvic ring. An update conference supported by the Club Bassin Cotyle (Pelvis-Acetabulum Club). Orthop Traumatol Surg Res. Feb 2013;99(Suppl 1):S77-86.

36. Nunn T, Cosker TD, Bose D, Pallister I. Immediate application of improvised pelvic binder as first step in extended resuscitation from life-threatening hypovolemic shock in conscious patients with unstable pelvic injuries. Injury. Jan 2007;38(1):125-8.

37. Knops SP, Van Lieshout EM, Spanjersberg WR, Patka P, Schipper IB Randomized clinical trial comparing pressure characteristics of pelvic circumferential compression devices in healthy volunteers. Injury. Oct 2011;42(10):1020-6.

38. Bonner TJ, Eardley WG, Newell N, Masouros S, Matthews JJ, Gibb I, et al. Accurate placement of a pelvic binder improves reduction of unstable fractures of the pelvic ring. J Bone Joint Surg Br. Nov 2011;93(11):1524-8.

39. Tan EC, van Stigt SF, van Vugt AB. Effect of a new pelvic stabilizer (T-POD $\AA$ ) on reduction of pelvic volume and haemodynamic stability in unstable pelvic fractures. Injury. Dec 2010;41(12):1239-43.

40. Totterman A, Madsen JE, Skaga NO, Roise O. Extraperitoneal pelvic packing: a salvage procedure to control massive traumatic pelvic hemorrhage. J Trauma. 2007;62:843e52.

41. Rossaint R, Bouillon B, Cerny V, Coats TJ, Duranteau J, Fernández-Mondéjar E, et al. The European guideline on management of major bleeding and coagulopathy following trauma: 4th ed. Crit Care. 12 Apr 2016;20:100.

42. Gaski IA, Barckman J, Naess PA, Skaga NO, Madsen JE, Kløw $\mathrm{NE}$, et al. Reduced need for extraperitoneal pelvic packing for severe pelvic fractures is associated with improved resuscitation strategies. J Trauma Acute Care Surg. Oct 2016;81(4):644-51.

43. Finan MA, Fiorica JV, Hoffman MS, Barton DP, Gleeson $\mathrm{N}$, Roberts WS, et al. Massive pelvic hemorrhage during gynecologic cancer surgery: "pack and go back." Gynecol Oncol. Sep 1996;62(3):390-5.

44. Roberts DJ, Bobrovitz N, Zygun DA, Ball CG, Kirkpatrick $\mathrm{AW}$, Faris PD, et al. Indications for use of thoracic, abdominal, pelvic, and vascular damage control interventions in trauma patients: A content analysis and expert appropriateness rating study. J Trauma Acute Care Surg. Oct 2015;79(4):568-79.

45. Lustenberger T, Wutzler S, Störmann P, Laurer H, Marzi I. The role of angio-embolization in the acute treatment concept of severe pelvic ring injuries. Injury. Oct 2015;46(Suppl 4):S33-8.

46. Li Q, Dong J, Yang Y, Wang G, Wang Y, Liu P, et al. Retroperitoneal packing or angioembolization for haemorrhage control of pelvic fractures. Quasi-randomized clinical trial of 56 haemodynamically unstable patients with Injury Severity Score $\geq 33$. Injury. Feb 2016;47(2):395-401.

47. Suzuki T, Smith WR, Moore EE. Pelvic packing or angiography: competitive or complementary? Injury. 2009;40:343-53. 